\title{
Eficacia de las alternativas de tratamiento para la mucositis periimplantaria
} Efficacy of treatment options for periimplant mucositis

\author{
ARDILA MEDINA CM* \\ GUZMÁN ZULUAGA IC**
}

Ardila Medina CM, Guzmán Zuluaga IC. Eficacia de las alternativas de tratamiento para la mucositis periimplantaria. Av Periodon Implantol. 2014; 26, 3: 141-146.

\begin{abstract}
RESUIMEN
Numerosos estudios han demostrado que la infección bacteriana juega un papel muy importante en el fracaso de los implantes dentales. Un desequilibrio huésped parásito en la interfase implante tejidos blandos inducen una prolongada reacción inflamatoria que ocasiona daño en los tejidos periimplantarios afectando la estabilidad del implante. Durante los primeros estados de inflamación ocurre un considerable daño tisular que exige la intervención del clínico con el fin de evitar lesiones irreversibles. Como en las enfermedades periodontales, se han propuesto varios tipos de terapias para reducir el número de especies patogénicas y mejorar así los parámetros clínicos de la mucositis periimplantar (MPI). De esta manera, la terapia básica para el tratamiento de la MPI comprende raspado mecánico, enjuagues con antisépticos, aplicación de sustancias quimioterapéuticas, aplicación local de antibióticos, o la combinación de estas alternativas. Con la poca evidencia disponible, parece que la terapia mecánica es efectiva en el tratamiento de la MPI.
\end{abstract}

PALABRAS CLAVE: Inflamación, mucositis periimplantaria, implantes, terapia.

\section{SUMMMARY}

Numerous studies have shown that bacterial infection plays the most important role in the late failures of dental implants. A host parasite imbalance, at the soft tissue-implant interface, inducing a prolonged inflammatory reaction, will result in periimplant tissue breakdown and possible implant failure. During the early stages of inflammation, tissue damage occurs that requires considerable intervention by the clinician to avoid irreversible damage. As in periodontal diseases, various therapies have been proposed to reduce the number of pathogenic species and improve the clinical parameters of peri-implant mucositis. In this way, the basic therapy for the treatment of MPI includes mechanical treatment, rinsing with antiseptics, application of chemotherapeutic substances, local application of antibiotics, or the combination of the above. With little evidence available, it appears that mechanical therapy alone is effective in the treatment of MPI.

KEY WORDS: Inflammation, periimplant mucositis, implants, therapy.

Fecha de recepción: 18 de julio 2009.

Fecha de aceptación: 21 de julio 2009. 


\section{INTRODUCCIÓN}

El éxito de los implantes dependen de la oseointegración y presencia de un contacto estrecho del epitelio y el tejido conectivo adyacente a la superficie del implante (1). La acumulación de bacterias sobre los implantes inician una respuesta inmune en la mucosa periimplantar caracterizada por el establecimiento de una lesión inflamatoria rica en leucocitos y estructuras vasculares (2). Las lesiones inflamatorias que se desarrollan en los tejidos alrededor de implantes incluyen dos entidades: mucositis periimplantaria (MPI) y periimplantitis. La MPI describe la presencia de inflamación en la mucosa de un implante sin signos de pérdida de soporte óseo, mientras que la periimplantitis se caracteriza principalmente por la existencia de pérdida ósea (3). Durante los primeros estados de inflamación ocurre un considerable daño tisular que exige la intervención del clínico con el fin de evitar lesiones irreversibles (3). Ericcson y cols. (4) evaluaron el efecto de la placa bacteriana sobre la encía de los dientes y la mucosa periimplantaria. El examen histológico de los dos tejidos mostró un infiltrado celular inflamatorio con muchas características en común, observándose mayor extensión apical del infiltrado celular en la mucosa periimplantaria que en la encía. Además, se ha observado gran similitud en la microflora subgingival en enfermedad periimplantaria y periodontal (5). Las terapias propuestas para el manejo de las enfermedades periimplantares se basan en la evidencia disponible para el tratamiento de la periodontitis, sin embargo el diseño de los implantes y el tratamiento de superficie de los mismos puede facilitar la formación de biopelícula si el implante se expone en la superficie bucal (6). De esta manera, la terapia básica para el tratamiento de la MPI comprende raspado mecánico, enjuagues con antisépticos, aplicación de sustancias quimioterapéuticas, aplicación local de antibióticos o la combinación de estas alter- nativas. El objetivo de este artículo es presentar la eficacia de las alternativas de tratamiento para el manejo de la MPI.

\section{TERAPIA MECÁNICA}

Dos estudios publicados recientemente y realizados por el mismo grupo de investigación evaluaron la eficacia de la terapia mecánica sobre la MPI $(7,8)$ (Tabla 1). En el primer trabajo, Máximo y cols. (7) evaluaron los efectos clínicos y microbiológicos de la terapia mecánica sobre la MPI. Se asignaron sujetos con al menos un implante dental a un grupo saludable $(n=10)$ o a un grupo con mucositis $(n=12)$. Los pacientes con MPI fueron tratados usando curetas de teflón y aire a presión con carbonato de sodio abrasivo. Al inicio del estudio y después de tres meses de realizado el tratamiento se evaluaron algunos índices clínicos (placa, sangrado, supuración, profundidad de sondaje y nivel de inserción clínica) y se tomaron muestras microbiológicas de la placa bacteriana de cada implante. A los tres meses post operatorios todos los parámetros clínicos mejoraron en los pacientes con mucositis $(p<0,05)$. Se observó una reducción en la profundidad de sondaje y en el nivel de inserción clínica de $1,3 \pm 1,2$ $\mathrm{mm}$ y $1,4 \pm 1,2 \mathrm{~mm}$ respectivamente. Después de tres meses de realizado el tratamiento también disminuyeron significativamente los niveles de Parvimonas micra, Fusobacterium nucleatum ss nucleatum, Porphyromons gingivalis, Treponema socranskii y las proporciones de complejo rojo $(p<0,05)$. En el segundo trabajo, Duarte y cols. (8) utilizando el mismo protocolo del estudio anterior evaluaron el efecto inmunológico de la terapia mecánica en pacientes con mucositis. Después de tres meses de tratamiento los niveles del factor de necrosis tumoral- $\alpha$ disminuyeron significativamente $(p<0,05)$ logrando resultados similares a los observados en el grupo de pacientes sanos.

\section{TABLA 1.- ESTUDIOS QUE EVALÚAN LA EFICACIA DE LA TERAPIA MECÁNICA SOBRE LA MUCOSITIS PERIIMPLANTAR}

\begin{tabular}{|c|c|c|}
\hline $\begin{array}{c}\text { Número de implantes } \\
\text { evaluados }\end{array}$ & $\begin{array}{c}\text { Tiempo de } \\
\text { seguimiento }\end{array}$ & \multicolumn{1}{|c|}{ Resultados obtenidos } \\
\hline 12 & 3 meses & $\begin{array}{l}\text { Reducción en la profundidad de sondaje y en el nivel de } \\
\text { inserción clínica (7). }\end{array}$ \\
\hline 12 & 3 meses & Disminución de los niveles de factor de necrosis tumoral- $\alpha(8)$. \\
\hline
\end{tabular}




\section{ANTISÉPTICOS}

Algunos investigadores han estudiado la efectividad de los antisépticos, como terapia única para el tratamiento de MPI (9-11) (Tabla 2). Lavigne y cols. (9) examinaron el efecto clínico, microbiológico e inmunológico de la irrigación con clorhexidina (CHX) sobre implantes con MPI. Un total de 30 implantes no adyacentes se asignaron aleatoriamente a uno de los tres grupos de evaluación (irrigación subgingival durante 15 segundos con $3 \mathrm{cc}$ de CHX al 0,12\%, irrigación subgingival durante 15 segundos con $3 \mathrm{cc}$ de solución salina y ninguna irrigación). Todos los parámetros se examinaron al inicio del tratamiento y a las dos, cinco y ocho semanas después de realizado. No se observó ninguna diferencia entre los grupos de tratamiento en ninguno de los parámetros clínicos, microbiológicos o inmunológicos. Felo y cols. (10) también compararon el efecto de la irrigación subgingival de CHX al 0,06\% (grupo experimental) con un enjuague de CHX al 0,12\% (grupo control), realizados una vez al día en pacientes con MPI. Los parámetros clínicos evaluados al inicio del estudio y a los tres meses de efectuado el tratamiento incluyeron niveles de placa y sangrado. La comparación entre los grupos mostró que la irrigación subgingival con CHX ocasionó una mayor reducción de los índices clínicos $(p<0,05)$. Sin embargo, los dos grupos presentaron disminución en los niveles de sangrado y placa al comparar estos parámetros clínicos al inicio del estudio con los obtenidos al tercer mes de realizado el tratamiento $(p<0,05)$. Ciancio y cols. (11) realizaron un ensayo clínico controlado para evaluar el efecto de un enjuague antiséptico (Listerine $($ ) sobre sujetos con MPI. Durante un período de tres meses evaluaron en 20 pacientes los niveles de placa bacteriana, sangrado gingival, profundidad de sondaje y nivel de inserción clínica. Como terapia adjunta a las medidas de higiene bucal rutinarias, los sujetos se enjuagaron dos veces al día durante 30 segundos con $20 \mathrm{ml}$ del enjuague asignado (grupo experimental: Listerine $\AA$; grupo placebo: hidro-alcohol al 5\%). A los tres meses, el grupo que usó el enjuague antiséptico presentó reducción estadísticamente significativa en los niveles de placa y sangrado gingival comparado con el grupo placebo, sin embargo, al evaluar la profundidad del sondaje y el nivel de inserción clínica no se observaron diferencias estadísticamente significativas entre los grupos.

\section{AGENTES QUIMIOTERAPÉUTICOS ADJUNTOS A LA TERAPIA MECÁNICA}

Un estudio realizado en un modelo animal (12) y tres efectuados en humanos (13-15) comparan la eficacia de la terapia mecánica con la combinación de agentes quimioterapéuticos y raspado mecánico en el tratamiento de la MPI. Trejo y cols. (12) realizaron un expe-

\section{TABLA 2.- ESTUDIOS QUE EVALÚAN LA EFICACIA DE LOS ANTISÉPTICOS COMO TERAPIA PARA EL TRATAMIENTO DE MUCOSITIS PERIIMPLANTAR}

\begin{tabular}{|c|c|c|}
\hline Grupos de tratamiento & $\begin{array}{c}\text { Tiempo de } \\
\text { seguimiento }\end{array}$ & Resultados obtenidos \\
\hline $\begin{array}{l}\text { - Irrigación subgingival de } \\
\text { CHX al 0,12\%. } \\
\text { - Irrigación subgingival de } \\
\text { solución salina. } \\
\text { - Ninguna irrigación. }\end{array}$ & 2 meses & $\begin{array}{l}\text { Ninguna diferencia entre los grupos en ningún parámetro } \\
\text { clínico, microbiológico o inmunológico (9). }\end{array}$ \\
\hline $\begin{array}{l}\text { - Irrigación subgingival de } \\
\text { CHX al 0,06\%. } \\
\text { - Enjuague de CHX al 0,12\%. }\end{array}$ & 3 meses & $\begin{array}{l}\text { La irrigación subgingival con CHX ocasionó mayor reduc- } \\
\text { ción de los índices clínicos }(p<0,05) \text {. } \\
\text { Los dos grupos presentaron disminución en los niveles de } \\
\text { sangrado y placa }(p<0,05)(10) \text {. }\end{array}$ \\
\hline $\begin{array}{l}\text { - Enjuague con Listerine }{ }^{\circledR} \text {. } \\
\text { - Enjuague con hidro-alcohol } \\
\text { al } 5 \% \text {. }\end{array}$ & 3 meses & $\begin{array}{l}\text { Listerine }{ }^{\circledR} \text { disminuyó niveles de placa y sangrado gingival } \\
\text { Ninguna diferencia en profundidad de sondaje y nivel de } \\
\text { inserción clínica (11). }\end{array}$ \\
\hline
\end{tabular}


rimento en nueve monos con el fin de evaluar clínica e histológicamente el efecto de diferentes alternativas terapéuticas. Dos implantes fueron ubicados en cada lado de la mandíbula. Se indujeron lesiones periimplantares permitiendo acumulación de placa durante seis semanas. Los animales de experimentación fueron asignados aleatoriamente a tres grupos: tratamiento mecánico solo (grupo A), terapia mecánica e irrigación local con CHX al 0,12\% más aplicación de CHX en gel al 0,2\% (grupo B) y un grupo control sin tratamiento (grupo C). Los promedios de profundidad de sondaje y los del nivel de inserción clínica de la MPI fueron: $3,5,3,7$ y $3,4 \mathrm{~mm}$, y $3,8,4,1$ y $3,9 \mathrm{~mm}$ para los grupos de tratamiento $\mathrm{A}, \mathrm{B}$ y $\mathrm{C}$, respectivamente. Los valores correspondientes después del tratamiento fue- ron: $1,7,2,1$ y $2,5 \mathrm{~mm}$, y $2,6,2,6$ y $3,1 \mathrm{~mm}$. No se observaron diferencias significativas entre los grupos de tratamiento A y B, pero si existieron entre estos dos grupos y el control $(p<0,01)$. Por otra parte, la proporción de inflamación encontrada en los tejidos del grupo control fue mayor al compararse con los grupos de tratamiento $(p<0,01)$. Un estudio similar, pero realizado en humanos, fue llevado a cabo por Porras y cols. (13), con el fin de determinar en 16 sujetos el efecto clínico del tratamiento con CHX sobre MPI, evaluando los niveles de placa, sangrado gingival, profundidad de sondaje y nivel de inserción clínica. Usando sondas de ADN también se examinó el efecto de la CHX sobre la microflora oral en la MPI. Después del examen inicial, los pacientes recibieron una profilaxis dental y fueron

\section{TABLA 3.- ESTUDIOS QUE EVALÚAN LOS AGENTES QUIMIOTERAPÉUTICOS ADJUNTOS A LA TERAPIA MECÁNICA}

\begin{tabular}{|c|c|c|}
\hline Grupos de tratamiento & $\begin{array}{l}\text { Tiempo de } \\
\text { seguimiento }\end{array}$ & Resultados obtenidos \\
\hline $\begin{array}{l}\text { - Tratamiento mecánico. } \\
\text { ción local con CHX al } \\
0,12 \% \text { mas aplicación de } \\
\text { CHX en gel al 0,2\%. } \\
\text { - Grupo control sin trata- } \\
\text { miento. }\end{array}$ & 3 meses & $\begin{array}{l}\text { Ninguna diferencia en la profundidad de sondaje y el nivel } \\
\text { de inserción clínica entre los grupos de tratamiento. } \\
\text { Diferencias entre los dos grupos de tratamiento y el control } \\
(p<0,01) \text {. } \\
\text { Mayor inflamación en los tejidos del grupo control }(p<0,01) \text { (12). }\end{array}$ \\
\hline $\begin{array}{l}\text { Terapia mecánica, instruc- } \\
\text { ción higiene oral, irriga- } \\
\text { ción local con CHX al } \\
0,12 \% \text { y aplicación tópica } \\
\text { de CHX en gel al 0,12\%. } \\
\text { Terapia mecánica e instruc- } \\
\text { ción en higiene bucal. }\end{array}$ & 3 meses & $\begin{array}{l}\text { Efectividad de los dos tratamientos para disminuir la pro- } \\
\text { fundidad de sondaje y mejorar los niveles de inserción. } \\
\text { Reducción de periodontopatógenos en los dos grupos (13). }\end{array}$ \\
\hline $\begin{array}{l}\text { - Terapia mecánica. } \\
\text { - Aplicación de ácido fosfó- } \\
\text { rico en gel al 35\%. }\end{array}$ & 5 meses & $\begin{array}{l}\text { Disminución del índice gingival y profundidad de sondaje } \\
\text { En el grupo control fue mayor la reducción del índice gin- } \\
\text { gival ( } p=0,03) \text {. } \\
\text { Disminución en los dos grupos en el número de unidades } \\
\text { formadoras de colonias. } \\
\text { Mayor reducción de bacterias con la limpieza química } \\
(p<0,05)(14) \text {. }\end{array}$ \\
\hline $\begin{array}{l}\text { - Raspado supra/subgingi- } \\
\text { val. } \\
\text { - Raspado supra/subgingival } \\
\text { más terapia adjunta con te- } \\
\text { traciclina. }\end{array}$ & 3 meses & $\begin{array}{l}\text { La terapia adjunta con tetraciclina redujo la hiperplasia de } \\
\text { la mucosa periimplantar, el sangrado y la profundidad al } \\
\text { sondaje (17). }\end{array}$ \\
\hline
\end{tabular}


asignados aleatoriamente al grupo experimental o al grupo control. El grupo experimental fue tratado con terapia mecánica, instrucción en higiene oral, irrigación local con CHX al 0,12\% y aplicación tópica de CHX en gel al 0,12\%. El grupo control recibió solamente terapia mecánica e instrucción en higiene bucal. En el tercer mes, los promedios de profundidad de sondaje y nivel de inserción clínica en el grupo experimental y en el grupo control fueron: $2,71 \pm 0,70 \mathrm{~mm}$ y $2,55 \pm 0,72$ $\mathrm{mm}$, y $2,30 \pm 1,50$ y $2,01 \pm 1,23 \mathrm{~mm}$ respectivamente $(p<0,05)$. Las dos alternativas de tratamiento fueron efectivas en la reducción de la MPI y la profundidad de sondaje, mejorando también los niveles de inserción. Adicionalmente, en los dos grupos y después de tres meses de evaluación, la mayoría de sitios estuvieron libres de periodontopatógenos.

Los resultados de este estudio confirman los hallados por la investigación en monos realizada por Trejo y col (12), en donde se apreció que el impacto de la CHX es casi insignificante cuando se compara con el efecto de la terapia mecánica sola. Strooker y cols. (14) realizaron un ensayo clínico con asignación aleatoria para comparar la efectividad de la terapia mecánica con la aplicación de un gel de ácido fosfórico al 35\%. Durante cinco meses se evaluaron 16 pacientes con MPI. Al grupo experimental se le aplicó en el surco periimplantar un gel ácido durante un minuto. Posteriormente, el surco se irrigó con agua durante 15 segundos. Al grupo control se le realizó terapia mecánica con curetas de fibra de carbón y copa de caucho. Los parámetros clínicos evaluados incluyeron índice de placa (15), índice gingival modificado (16) y profundidad de sondaje. También se realizó una evaluación microbiológica antes e inmediatamente después de cada tratamiento. En el quinto mes de evaluación se disminuyeron los promedios de índice gingival (16) y profundidad de sondaje, sin embargo, en el grupo control la reducción del índice gingival fue significativamente mayor (16) $(p=0,03)$. En las dos modalidades de tratamiento se observó una disminución en el número de unidades formadoras de colonias, pero se obtuvo más reducción con la limpieza química $(p<0,05)$. Finalmente, Schenk y cols. (17) evaluaron la eficacia de la aplicación controlada de tetraciclina tópica en el tratamiento de MPI. Se incluyeron en el estudio ocho pacientes con al menos dos implantes que mostraran signos clínicos de MPI. Todos los implantes se sometieron a raspado supra y subgingival, y la mitad de ellos recibieron terapia adjunta con tetraciclina (grupo experimental). Se evaluaron los parámetros clínicos en la cuarta semana y en el tercer mes de tratamiento. En el grupo experimental se observó una disminución marcada de la hi- perplasia de la mucosa periimplantar y en el sangrado y profundidad al sondaje. En los dos implantes que sirvieron como control no se observó efecto de la terapia sobre la mucosa periimplantar. En los dos grupos, se disminuyó el índice de placa (15) a las cuatro semanas pero regresó a su nivel base después del tercer mes, sin embargo, la profundidad de sondaje y el nivel de inserción clínica permanecieron sin cambio durante todo el ensayo clínico. Es importante destacar que este estudio no presentó el tamaño de muestra necesario para alcanzar suficiente poder estadístico que permitiera evaluar la eficacia de las dos alternativas de tratamiento.

\section{CONCLUSIONES}

Con la poca evidencia disponible, parece que la terapia mecánica sola es efectiva en el tratamiento de la MPI. El tratamiento quimioterapéutico adjunto a la terapia mecánica puede mejorar los resultados del tratamiento, sin embargo, se requieren ensayos clínicos bien diseñados, con tamaños de muestra suficiente que permitan evaluar la adición de agentes quimioterapéuticos a los protocolos de terapia mecánica en el tratamiento de mucositis periimplantar.

\section{BIBLIOGRAFÍA}

1. Listgarten MA, Lang NP, Schroeder HE, Schroeder A. Periodontal tissues and their counterparts around endosseous implants. Clin Oral Implants Res 1991;2:1-19.

2. Zitzmann NU, Abrahamsson I, Berglundh T, Lindhe J. Soft tissue reactions to plaque formation at implant abutments with different surface topography. An experimental study in dogs. J Clin Periodontol 2002;29: 456-61.

3. Zitzmann NU, Berglundh T. Definition and prevalence of peri-implant diseases. J Clin Periodontol 2008;35 (Suppl. 8):286-91.

4. Ericsson I, Berglundh T, Marinello C, Liljenberg B, Lindhe J. Long-standing plaque and gingivitis at implants and teeth in the dog. Clin Oral Implants Res 1992;3:99103.

5. Mombelli A, van Oosten MA, Schurch E Jr, Land NP. The microbiota associated with successful or failing osseointegrated titanium implants. Oral Microbiol Immunol 1987;2:145-51. 
6. Pongnarisorn NJ, Gemmell E, Tan AES, Henry PJ, Marshall RI, Seymour GJ. Inflammation associated with implants with different surface types. Clin Oral Impl Res 2007; 18:114-25.

7. Máximo MB, de Mendonça AC, Renata Santos V, Figueiredo LC, Feres M, Duarte PM. Short-term clinical and microbiological evaluations of peri-implant diseases before and after mechanical anti-infective therapies. Clin Oral Implants Res 2009;20:99-108.

8. Duarte PM, de Mendonça AC, Máximo MB, Santos VR, Bastos MF, Nociti FH. Effect of anti-infective mechanical therapy on clinical parameters and cytokine levels in human peri-implant diseases. J Periodontol 2009;80: 234-43.

9. Lavigne SE, Krust-Bray KS, Williams KB, Killoy WJ, Theisen F. Effects of subgingival irrigation with chlorhexidine on the periodontal status of patients with HA-coated integral dental implants. Int J Oral Maxillofac Implants 1994; 9:156-62.

10. Felo A, Shibly O, Ciancio SG, Lauciello FR, Ho A. Effects of subgingival chlorhexidine irrigation on peri-implant maintenance. Am J Dent 1997;10:107-10.

11. Ciancio SG, Lauciello F, Shibly O, Vitello M, Mather M. The effect of an antiseptic mouthrinse on implant maintenance: plaque and peri-implant gingival tissues. J Periodontol 1995;66:962-5.

12. Trejo PM, Bonaventura G, Weng D, Caffesse RG, Bragger U, Lang NP. Effect of mechanical and antiseptic therapy on peri-implant mucositis: an experimental study in monkeys. Clin Oral Implants Res 2006;17:294-304.

13. Porras R, Anderson GB, Caffesse R, Narendran S, Trejo PM. Clinical response to 2 different therapeutic regimens to treat peri-implant mucositis. J Periodontol 2002;73:1 118-25.

14. Strooker H, Rohn S, Van Winkelhoff AJ. Clinical and microbiologic effects of chemical versus mechanical cleansing in professional supportive implant therapy. Int J Oral Maxillofac Implants 1998;13:845-50.

15. Silness J, Löe H. Periodontal disease in pregnancy. II. Correlation between oral hygiene and periodontal condition. Acta Odontol Scand 1964;24:747-59.

16. Löe H, Silness J. Periodontal disease in pregnancy. I. Prevalence and severity. Acta Odontol Scand 1963;21: 533-51.

17. Schenk G,Flemmig TF, BetzT, Reuther J, Klaiber B. Controlled local delivery of tetracycline $\mathrm{HCl}$ in the treatment of periimplant mucosal hyperplasia and mucositis. A controlled case series. Clin Oral Implants Res 1997;8:427-33.

\section{CORRESPONDENCIA}

Carlos Martín Ardila Medina

Carrera 47 No. 20 sur 46

Envigado Antioquía

Colombia

Correo electrónico: martinardila@gmail.com 